\title{
Out-of-equilibrium dynamics and thermalization of string order
}

\author{
Leonardo Mazza, ${ }^{1}$ Davide Rossini, ${ }^{1}$ Manuel Endres, ${ }^{2}$ and Rosario Fazio ${ }^{1}$ \\ ${ }^{1}$ NEST, Scuola Normale Superiore and Istituto Nanoscienze-CNR, I-56126 Pisa, Italy \\ ${ }^{2}$ Max-Planck-Institut für Quantenoptik, D-85748 Garching, Germany
}

(Received 11 November 2013; revised manuscript received 3 May 2014; published 11 July 2014)

\begin{abstract}
We investigate the equilibration dynamics of string order in one-dimensional quantum systems. After initializing a spin- 1 chain in the Haldane phase, the time evolution of nonlocal correlations following a sudden quench is studied by means of matrix-product-state-based algorithms. Thermalization occurs only for scales up to a horizon growing at a well defined speed, due to the finite maximal velocity at which string correlations can propagate, related to a Lieb-Robinson bound. The persistence of string ordering at finite times is nontrivially related to symmetries of the quenched Hamiltonian. A qualitatively similar behavior is found for the string order of the Mott insulating phase in the Bose-Hubbard chain. This paves the way towards an experimental testing of our results in present cold-atom setups.
\end{abstract}

DOI: 10.1103/PhysRevB.90.020301

Introduction. Characterizing the long-time properties of many-body systems evolving according to a unitary time evolution is a long-standing and fascinating task [1-4]. Because of special physical importance, the equilibration of Landau order parameters and of two-point correlators has deserved almost unique attention. It is by now assessed, however, that not every quantum phase of matter is amenable for a description in terms of local observables. The peculiarity of topological order only emerges via the study of nonlocal operators [5-7].

Triggered by impressive experimental progress on ultracold atomic gases, where both the initial state and the Hamiltonian dynamics can be engineered with unprecedented accuracy [8-14], recent years have witnessed a burst in the theoretical understanding of the equilibration of closed systems [15-17]. When the dynamics is governed by a nonintegrable Hamiltonian, such that the energy is the only nontrivial conserved quantity, the features of a canonical ensemble are expected to emerge locally in the steady state [18-26]. The occurrence of thermalization for nonlocal order, instead, cannot be predicted via this picture, and stands as an intriguing open problem. The literature on this topic mainly focused on the equilibration dynamics of entropy measures for topological order [27-30].

Here we study the time evolution after a global quantum quench of a string operator for a one-dimensional system. Since the string can extend over a macroscopic part of the chain, its dynamics does not simply fit into the picture of a system acting as the bath of one of its parts. We start discussing the string order (SO) in a spin-1 chain [6]. After quenching the system within or outside the Haldane phase, string correlations reveal thermalization on short lengths but the finite velocity at which they propagate, related to a Lieb-Robinson (LR) bound, prevents this from occurring for longer lengths. Moreover, a symmetry analysis reveals the existence of Hamiltonians destroying the SO abruptly. The possibility to experimentally access string operators with cold atoms [31] allows a direct test of our predictions. To this aim we conclude by analyzing quenches within the Mott insulating phase in the Bose-Hubbard (BH) chain, where this type of experiment is most likely to be performed.

Quantum quenches and string order. As a paradigm to study out-of-equilibrium SO, we consider the Haldane phase in
PACS number(s): 75.10.Pq, 05.30.Jp, 05.70.Ln

spin-1 infinite chains, a symmetry-protected topological phase [32,33] characterized by SO [34]:

$$
\mathcal{O}^{\alpha} \doteqdot \lim _{|i-j| \rightarrow \infty}\left\langle\hat{\mathcal{O}}_{i, j}^{\alpha}\right\rangle \neq 0
$$

where $\hat{\mathcal{O}}_{i, j}^{\alpha} \doteqdot \hat{S}_{i}^{\alpha} \otimes e^{i \pi \hat{S}_{i+1}^{\alpha}} \cdots e^{i \pi \hat{S}_{j-1}^{\alpha}} \otimes \hat{S}_{j}^{\alpha}$ and $\hbar \hat{S}_{j}^{\alpha}$ is the $\alpha=x, y, z$ component of the spin operator at the $j$ th site. SO signals the presence of a "dilute" antiferromagnetic order where oriented spins $S_{i}^{\alpha}= \pm 1$ spatially alternate, although they can be separated by arbitrary-length strings of $S_{i}^{\alpha}=0$ spins [Fig. 1(a)]. Spin-1 chains can also effectively mimic the physics of cold bosonic gases trapped in one-dimensional (1D) optical lattices, where the Haldane phase may appear [7,35].

The ground state of the Affleck-Kennedy-Lieb-Tasaki (AKLT) model in the $\sum_{i}\left\langle\hat{S}_{i}^{z}\right\rangle=1$ magnetization sector, $\left|\Psi_{0}\right\rangle$, is taken as the initial state [36]. It belongs to the Haldane phase and admits an exact matrix-product-state (MPS) description with bond link $D=2$ [37], which lowers technical intricacies and allows the investigation of longer time evolutions. Next, $\left|\Psi_{0}\right\rangle$ is let free to evolve unitarily according to the Hamiltonian $\hat{\mathcal{H}}$, such that $|\Psi(t)\rangle \doteqdot e^{-i \hat{\mathcal{H}} t / \hbar}\left|\Psi_{0}\right\rangle$. In the following, we characterize the SO dynamics by investigating the time and length dependence of $\left\langle\hat{\mathcal{O}}_{i, i+\ell}^{\alpha}\right\rangle(t)$, where $\langle\cdot\rangle(t)$ denotes the expectation value over $|\Psi(t)\rangle$. We employ several numerical algorithms based on MPS [38,39]: $|\Psi(t)\rangle$ is computed with a time-evolving block-decimation technique [40], whereas thermal properties are obtained with the ancilla method [41].

String operators in spin-1 chains. We start considering the antiferromagnetic spin-1 $X X Z$ model:

$$
\hat{\mathcal{H}}=J \sum_{i}\left(\hat{S}_{i}^{x} \hat{S}_{i+1}^{x}+\hat{S}_{i}^{y} \hat{S}_{i+1}^{y}+\Delta \hat{S}_{i}^{z} \hat{S}_{i+1}^{z}\right),
$$

with $J>0$. For simplicity, we assume $\Delta>0$ : In this case, when $\Delta<\Delta_{c}\left(\Delta_{c} \sim 1.18\right)$ the ground state is in the Haldane phase, whereas for $\Delta>\Delta_{c}$ it presents Néel order [42]. The model is integrable only in the classical limit $\Delta \rightarrow \infty$.

We first discuss the case $\Delta=0.2$ (Fig. 1). For any finite $t>0$, the state displays SO, because the expectation value of longer string operators saturates to a nonzero plateau as a function of length. The dynamics of the string operator in the $x$ direction, $\left\langle\hat{\mathcal{O}}_{i, i+\ell}^{x}\right\rangle(t)$, exhibits two regions separated by a length scale $L_{\mathrm{th}}(t)$. For longer strings, $\ell \gg L_{\mathrm{th}}(t)$, 
(a)
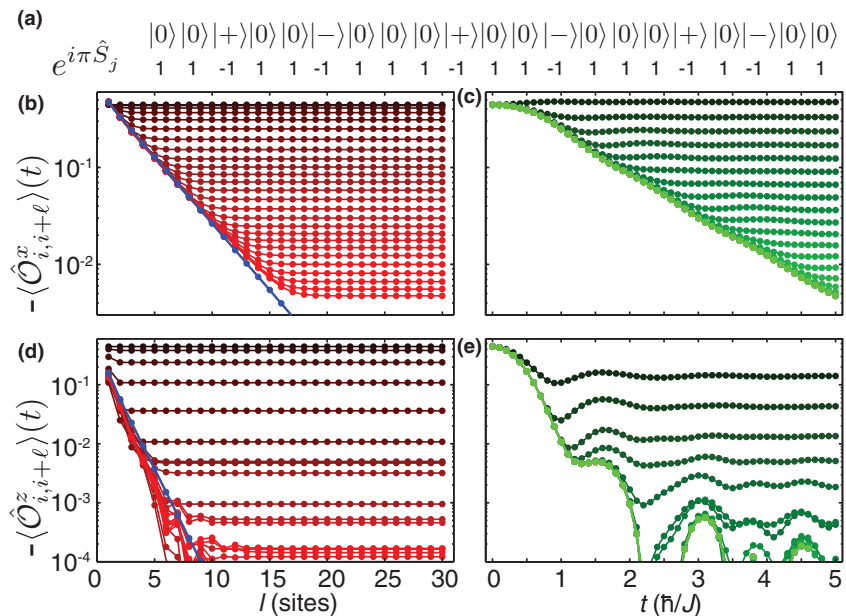

FIG. 1. (Color online) (a) Sketch of a product state featuring the dilute antiferromagnetic order detected by $\hat{\mathcal{O}}_{i, j}^{z}$. (b)-(e) String correlations for a quench of $\left|\Psi_{0}\right\rangle$ with Hamiltonian (2), $\Delta=0.2$. (b) $\left\langle\hat{\mathcal{O}}_{i, i+\ell}^{x}\right\rangle(t)$ as a function of the length $\ell$. Each line refers to a different time, ranging from $t=0 \hbar / J$ (dark red) to $t=5.0 \hbar / J$ (bright red), with spacing $0.2 \hbar / J$. The blue line represents string correlations of the thermal state $\hat{\rho}_{\text {th }}(\beta)$, with $\beta=1.6 J^{-1}$. (c) $\left\langle\hat{\mathcal{O}}_{i, i+\ell}^{x}\right\rangle(t)$ as a function of $t$. Each line refers to a different $\ell$, ranging from $\ell=1$ (dark green) to $\ell=30$ (bright green), with spacing 1 . (d), (e) $\left\langle\hat{\mathcal{O}}_{i, i+\ell}^{z}\right\rangle(t)$ with color coding and axes as in (b) and (c), respectively.

such operator is exponentially close to its infinite-length value: $\left\langle\hat{\mathcal{O}}_{i, i+\ell}^{x}\right\rangle(t) \approx \mathcal{O}^{x}(t)$. Oppositely, for shorter strings, $\ell \ll L_{\mathrm{th}}(t)$, it is close to its infinite-time value: $\left\langle\hat{\mathcal{O}}_{i, i+\ell}^{x}\right\rangle(t) \approx$ $\lim _{t \rightarrow \infty}\left\langle\hat{\mathcal{O}}_{i, i+\ell}^{x}\right\rangle(t)$. As we discuss below, the long-string region can be understood in terms of a LR bound, while short strings suggest the appearance of a thermal region growing with time. Data for the string in the $z$ direction, $\left\langle\hat{\mathcal{O}}_{i, i+\ell}^{z}\right\rangle(t)$, indicate a similar behavior, which is, however, less conclusive due to superimposed damped oscillations in time. Note that $\left\langle\hat{\mathcal{O}}_{i, i+\ell}^{z}\right\rangle(t)$ is typically an order of magnitude smaller than $\left\langle\hat{\mathcal{O}}_{i, i+\ell}^{x}\right\rangle(t)$, analogously to what happens for the ground state of Hamiltonian (2) for $\Delta=0.2$ [43].

The long-string behavior is best understood via the following key observation. For Hamiltonian (2), string correlations satisfy a LR bound $[44,45]$ :

$$
\left|\left\langle\hat{\mathcal{O}}_{i, i+\ell}^{\alpha}\right\rangle(t)-\mathcal{O}^{\alpha}(t)\right|<C e^{-(\ell-v t) / \xi} .
$$

Equation (3) follows directly from the Kennedy-Tasaki transformation $[46,47], \hat{\mathcal{U}}_{\mathrm{KT}}=\prod_{j<k} \exp \left(i \pi \hat{S}_{j}^{z} \hat{S}_{k}^{x}\right)$, which is unitary and nonlocal. This dual transformation maps (2) into another local model, which is ferromagnetic and for $0 \leqslant \Delta \leqslant \Delta_{c}$ has four symmetry-broken magnetic ground states. String operators are mapped to two-point operators: $\hat{\mathcal{U}}_{\mathrm{KT}} \hat{\mathcal{O}}_{i, j}^{\alpha} \hat{\mathcal{U}}_{\mathrm{KT}}^{\dagger}=$ $\hat{S}_{i}^{\alpha} \hat{S}_{j}^{\alpha}$. In this dual picture, spin-spin correlations obey the LR bound $\left|\left\langle\hat{S}_{i}^{\alpha} \hat{S}_{i+\ell}^{\alpha}\right\rangle(t)-\left\langle\hat{S}_{i}^{\alpha}\right\rangle\left\langle\hat{S}_{i+\ell}^{\alpha}\right\rangle(t)\right|<C e^{-(\ell-v t) / \xi}$ [45]. Indeed, take as the initial state $\hat{\mathcal{U}}_{\mathrm{KT}}^{\dagger}|\Psi\rangle$ : The insertion of $\hat{\mathcal{U}}_{\mathrm{KT}} \hat{\mathcal{U}}_{\mathrm{KT}}^{\dagger}$ in every expectation value and the fact that $\mathcal{O}^{\alpha} \doteqdot \lim _{|i-j| \rightarrow \infty}\left\langle\hat{\mathcal{O}}_{i, j}^{\alpha}\right\rangle=\left\langle\hat{\mathcal{U}}_{\mathrm{KT}}^{\dagger} \hat{S}_{i}^{\alpha} \hat{\mathcal{U}}_{\mathrm{KT}}\right\rangle^{2}$ yield Eq. (3). In general, a LR bound for string operators can be derived whenever there is a mapping to a dual model such that the string
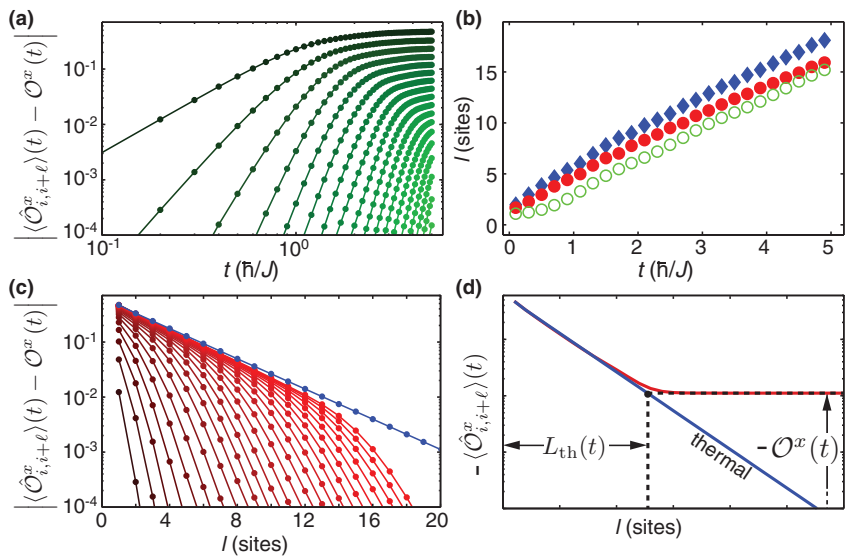

FIG. 2. (Color online) String correlations for a quench with Hamiltonian (2), $\Delta=0.2$. (a) Subtracted string correlations $\left|\left\langle\hat{\mathcal{O}}_{i, i+\ell}^{x}\right\rangle(t)-\mathcal{O}^{x}(t)\right|$ as a function of time. Color code as in Fig. 1(c). (b) Spreading of string correlations. Space-time dependence of $t_{F_{0}}(\ell)$ for $F_{0}=10^{-4}$ (blue diamonds) and $10^{-3}$ (red dots). Time dependence of $L_{\mathrm{th}}(t)$ (green empty circles). (c) Subtracted string correlations $\left|\left\langle\hat{\mathcal{O}}_{i, i+\ell}^{x}\right\rangle(t)-\mathcal{O}^{x}(t)\right|$ as a function of length. Color code as in Fig. 1(b). The blue line represents string correlations of the thermal state $\hat{\rho}_{\mathrm{th}}(\beta)$, with $\beta=1.6 J^{-1}$. (d) Illustration of the construction of $L_{\mathrm{th}}(t)$.

operator is mapped to a two-site correlator obeying a LR bound [48].

In Fig. 2, we study the subtracted correlations of Eq. (3). A causal-cone effect is observed. Correlations are initially zero and increase more slowly the longer the considered string is [panel (a)]. The dispersion relation of the cone can be extracted by identifying the time $t_{F_{0}}(\ell)$ satisfying $\left|\left\langle\mathcal{O}_{i, i+\ell}^{x}\right\rangle(t)-\mathcal{O}^{\alpha}(t)\right|=F_{0}$. A linear space-time dependence appears, $t_{F_{0}}(\ell) \sim \ell / v_{\mathrm{LR}}$, signaling a ballistic spreading of string correlations for short times, even if the system is nonintegrable [panel (b)]. Panel (c) points out that the value $\mathcal{O}^{x}$ is approached exponentially as a function of length, as predicted in Eq. (3).

For short strings, the behavior of $\left\langle\hat{\mathcal{O}}_{i, i+\ell}^{x}\right\rangle(t)$ appears to be well captured by a thermal ensemble [49], $\hat{\rho}_{\text {th }}(\beta)=e^{-\beta \hat{H}} / \mathcal{Z}$ ( $\mathcal{Z}$ is the partition function), whose effective temperature $\beta$ is defined by $\left\langle\Psi_{0}|\hat{\mathcal{H}}| \Psi_{0}\right\rangle=\operatorname{tr}\left[\hat{\mathcal{H}} \hat{\rho}_{\text {th }}(\beta)\right]$. As shown in Fig. 1, the SO of $|\Psi(t)\rangle$ can be characterized in this way for strings up to length $\ell=10$. Note that $\hat{\mathcal{O}}_{i, i+\ell}^{\alpha}$ probes the properties of a multisite region, thus providing information not included in any two-point observable. The typical length scale $L_{\text {th }}(t)$ separating the thermal from the LR region, can be defined according to $\operatorname{tr}\left[\hat{\mathcal{O}}_{i, i+L_{\text {th }}(t)}^{x} \hat{\rho}_{\text {th }}(\beta)\right]=\mathcal{O}^{x}(t)$, where $L_{\text {th }}(t)$ is the length at which the thermal value of the string operator crosses the infinite-length value of the dynamically evolved string operator [see Fig. 2(d)]. Figure 2(b) shows a linear space-time dependence $L_{\mathrm{th}}(t) \sim v_{\mathrm{th}} t$, compatible to $v_{\mathrm{LR}} t_{F_{0}}$.

We now extend the previous analysis to other values of $\Delta$. The presence of an increasing region with size $v t$, where string correlations $\left\langle\hat{\mathcal{O}}_{i, j}^{x}\right\rangle(t)$ are thermal-like, approximately holds for $\Delta \lesssim 1.2$ (see Fig. 3). For larger values of $\Delta$, an oscillatory behavior sets in, complicating the short-time evolution. From our data, it is not possible to tell whether this is due to approaching the integrable point $\Delta \rightarrow+\infty$, or 


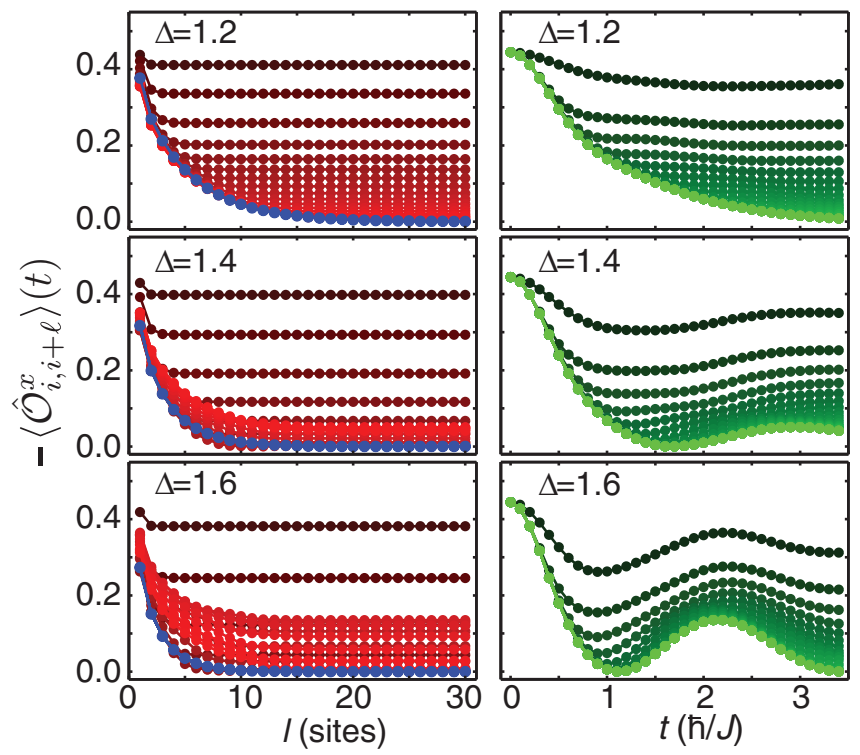

FIG. 3. (Color online) String correlations $\left\langle\mathcal{O}_{i, i+\ell}^{x}\right\rangle(t)$ for a quench with Hamiltonian (2) for $\Delta=1.2,1.4$, and 1.6, as a function of length $\ell$ (left panels) and time $t$ (right panels). Left panels: Each line refers to a different time, ranging from $t=0 \hbar / J$ (dark red) to $t=3.4 \hbar / J$ (bright red), with spacing $0.2 \hbar / J$. Blue lines represent string correlations of the thermal states $\hat{\rho}_{\mathrm{th}}(\beta)$, with $\beta=1.92,1.48$, and $1.2 J^{-1}$, respectively. Right panels: Each line refers to a different length, ranging from $\ell=1$ (dark green) to $\ell=30$ (bright green), with spacing 1 .

the phase transition at $\Delta_{c} \sim 1.18$. Note that for all our cases $\beta / E_{\text {gap }}<10$, where $E_{\text {gap }}$ is the second energy gap for $L=60$ computed for $\sum_{i}\left\langle\hat{S}_{i}^{z}\right\rangle=0$. (Two degenerate ground states are expected in the thermodynamic limit.)

A natural explanation of our data goes as follows: The density matrix $\hat{\rho}_{S}=\operatorname{tr}_{\text {chain } / S}[|\Psi(t)\rangle\langle\Psi(t)|]$ of a region $S$ of size $v t$ appears to be thermal. This interpretation is corroborated by our study of $\hat{\rho}_{S}$ for regions up to three sites and by the study of $\left\langle\hat{S}_{i}^{x} \hat{S}_{j}^{x}\right\rangle(t)$ correlations (not shown). The behavior of $\left\langle\hat{\mathcal{O}}_{i, i+\ell}^{z}\right\rangle(t)$ does not fit immediately in this framework [see Figs. 1(d) and 1(e)]. Even if short strings equilibrate (with superimposed damped oscillations) within accessible times, this happens more slowly than for $\left\langle\hat{\mathcal{O}}_{i, i+\ell}^{x}\right\rangle(t)$, hinting at the possibility that different internal degrees of freedom equilibrate in significantly different ways.

Symmetry protection. In the previous discussion we stressed that $|\Psi(t)\rangle$ exhibits SO for every accessible $t$, meaning that the long-string limit in Eq. (1) is different from zero. This is not always the case and depends on the symmetries of $\hat{\mathcal{H}}$. We now prove a necessary and sufficient condition on $\hat{\mathcal{H}}$ for $|\Psi(t)\rangle$ to display SO at infinitesimally short times.

We recall that a necessary condition for a state $|\Phi\rangle$ to possess the SO of Eq. (1) is to be symmetric under the action of a global $e^{i \pi \sum_{j} \hat{S}_{j}^{\alpha}}$ transformation: $e^{i \pi \sum_{j} \hat{S}_{j}^{\alpha}}|\Phi\rangle=$ $e^{i \theta_{\alpha}}|\Phi\rangle$ [50]. We first investigate necessary and sufficient conditions for $e^{i \pi \sum_{j} \hat{S}_{j}^{\alpha}}|\Psi(t)\rangle=|\Psi(t)\rangle, \forall t$. We focus on the Abelian $\mathbb{D}_{2}$ group of $\pi$ rotations along three orthogonal axes $e^{i \pi \sum_{j} \hat{S}_{j}^{\alpha}}, \alpha=x, y, z$. Note that $e^{i \theta_{\alpha}}=1 \forall t$ for $\alpha=x, z$ because $e^{i \pi \sum_{j} \hat{S}_{j}^{\alpha}}\left|\Psi_{0}\right\rangle=\left|\Psi_{0}\right\rangle$, time evolution is continuous, and the eigenvalues of $e^{i \pi \sum_{j} \hat{S}_{j}^{\alpha}}$ are discrete.

We begin by considering an infinitesimal time $\delta t$, so that $e^{-i \hat{\mathcal{H}} \delta t / \hbar} \approx \mathbb{I}-i(\delta t / \hbar) \hat{\mathcal{H}}$. Let us decompose $\hat{\mathcal{H}}=\hat{\mathcal{H}}_{\mathrm{e}}+$ $\hat{\mathcal{H}}_{\mathrm{o}}, \hat{\mathcal{H}}_{\mathrm{e}}$ being the symmetric part of the Hamiltonian: $e^{i \pi \sum_{j} \hat{S}_{j}^{\alpha}} \hat{\mathcal{H}}_{\mathrm{e}} e^{-i \pi \sum_{j} \hat{S}_{j}^{\alpha}}=\hat{\mathcal{H}}_{\mathrm{e}}$ for $\alpha=x, z$, and $\hat{\mathcal{H}}_{\mathrm{o}}$ the rest. Clearly, when the Hamiltonian is symmetric $\left(\hat{\mathcal{H}}_{\mathrm{o}}=0\right),|\Psi(t)\rangle$ is $\mathbb{D}_{2}$ symmetric. This is true also in the presence of nonsymmetric contributions $\left(\hat{\mathcal{H}}_{\mathrm{o}} \neq 0\right)$. At first order in $\delta t$, this is equivalent to $\hat{\mathcal{H}}_{\mathrm{o}}\left|\Psi_{0}\right\rangle=0$ because $\hat{\mathcal{H}}_{\mathrm{o}}$ connects $\left|\Psi_{0}\right\rangle$ only to states which are not left invariant by the action of every element of the $\mathbb{D}_{2}$ group. A full series expansion of $e^{-i \hat{\mathcal{H}} t / \hbar}$ yields that the $\mathbb{D}_{2}$ symmetry of the state $|\Psi(t)\rangle$ is equivalent to

$$
\hat{\mathcal{H}}_{\mathrm{o}}\left(\hat{\mathcal{H}}_{\mathrm{e}}\right)^{n}\left|\Psi_{0}\right\rangle=0, \quad \forall n \in \mathbb{N}_{0} .
$$

Since $\mathbb{D}_{2}$ symmetry is necessary for SO, (4) is a necessary condition for $|\Psi(t)\rangle$ to display SO.

To show that it is also sufficient, we prove continuity of $\mathcal{O}^{\alpha}(t)$ as a function of $t \quad\left(\mathcal{O}^{\alpha} \neq 0\right.$ at $\left.t=0\right)$. Let us observe that $\left\langle\hat{\mathcal{O}}_{i, j}^{\alpha}\right\rangle(\delta t)=\left\langle\hat{\mathcal{O}}_{i, j}^{\alpha}\right\rangle(0)+i(\delta t / \hbar)$ $\left\langle\Psi_{0}\left|\left[\hat{\mathcal{H}}_{\mathrm{e}}, \hat{\mathcal{O}}_{i, j}^{\alpha}\right]\right| \Psi_{0}\right\rangle+o\left(\delta t^{2}\right)$ where $\hat{\mathcal{H}}_{\mathrm{o}}$ is discarded because of (4). We assume $\hat{\mathcal{H}}_{\mathrm{e}}$ to be short ranged: $\hat{\mathcal{H}}_{\mathrm{e}}=\sum_{j} \hat{h}_{\mathrm{e}, j}$, each $\hat{h}_{\mathrm{e}, j}$ having support on $k$ neighboring spins. Thus the operator $\left[\hat{\mathcal{H}}_{\mathrm{e}}, \hat{\mathcal{O}}_{i, j}^{\alpha}\right]$ has support at most on $2 k$ spins centered around $i$ and around $j$, and is bounded even for $|i-j| \rightarrow \infty$. Using (4) one can prove that terms of order $(\delta t / \hbar)^{n}$ for $n>1$ are bounded as well. Continuity follows, so that Eq. (4) is equivalent to the presence of $\mathrm{SO}$ at finite times.

When condition (4) is violated, SO is lost abruptly, namely, in infinitesimal time: Even if at $t=0$ the limit in Eq. (1) is different from zero, at time $\delta t$ it becomes exactly zero. Notwithstanding the continuity of the time-evolution operator, the long-string limit can make the SO parameter discontinuous in time.

Remarkably, SO is more resilient to perturbations in a dynamical context than in a static one. Indeed, a necessary condition for the ground state of $\hat{\mathcal{H}}$ to display SO in Eq. (1) is that $\hat{\mathcal{H}}$ commutes with $e^{i \pi \sum_{j} \hat{S}_{j}^{\alpha}}$ [32]. However, SO can withstand a quench by a nonsymmetric Hamiltonian, as long as (4) holds. An example is the spin-1 $X X Z$ model (2) with an additional magnetic field along $z$. Equation (4) enforces that $|\Psi(t)\rangle$ is in the kernel of $\hat{\mathcal{H}}_{\mathrm{o}}$, so that effectively it can be computed only considering $\hat{\mathcal{H}}_{\mathrm{e}}$.

String operators in the Bose-Hubbard model. The out-ofequilibrium study of string correlations is within immediate experimental reach. Ultracold bosonic atoms prepared in 1D tubes with a deep optical lattice along the system direction can be described by a BH model

$$
\hat{\mathcal{H}}_{\mathrm{BH}}=-J \sum_{j}\left(\hat{a}_{j}^{\dagger} \hat{a}_{j+1}+\text { H.c. }\right)+\frac{U}{2} \sum_{j} \hat{n}_{j}\left(\hat{n}_{j}-1\right),
$$

where $\hat{a}_{j}^{\dagger}$ creates one boson on site $j, \hat{n}_{j}=\hat{a}_{j}^{\dagger} \hat{a}_{j}, J$ is the hopping rate, and $U$ the interaction strength. For $U \gg J$ the model displays a Mott insulating (MI) phase characterized by the string $\hat{\mathcal{O}}_{i, j}^{(\mathrm{BH})} \doteqdot e^{i \pi \delta \hat{n}_{i}} \otimes e^{i \pi \delta \hat{n}_{i+1}} \cdots e^{i \pi \delta \hat{n}_{j-1}} \otimes e^{i \pi \delta \hat{n}_{j}}, \delta \hat{n}_{j}$ being the fluctuation over the average boson density at site $j$. String correlations for up to nine sites have been detected 

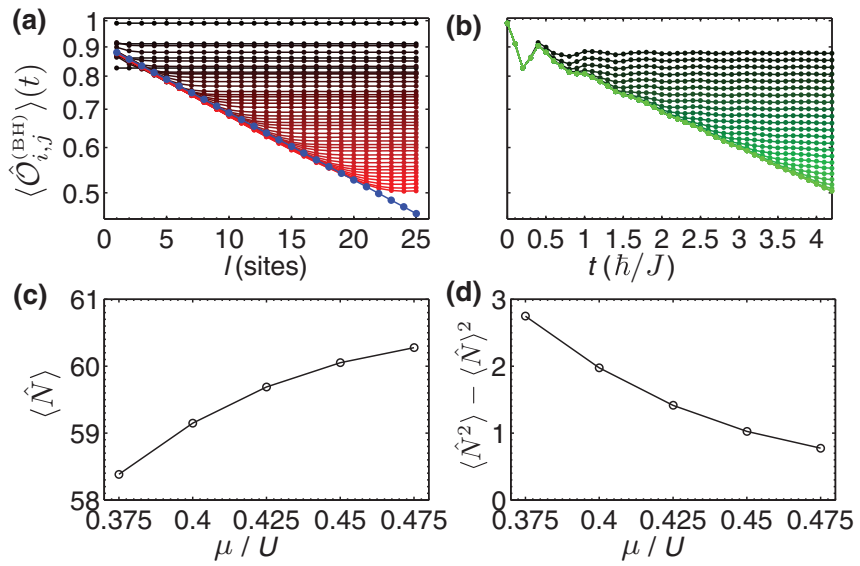

FIG. 4. (Color online) String correlations $\left\langle\hat{\mathcal{O}}_{i, i+\ell}^{(\mathrm{BH})}\right\rangle(t)$ as a function of $\ell$ for a quench with Hamiltonian (5), from $U_{0}=40 \mathrm{~J}$ to $U=15 J$. (a) Each line refers to a different time, ranging from $t=0$ $\hbar / J$ (dark red) to $t=4.2 \hbar / J$ (bright red) with spacing $0.1 \hbar / J$. The blue line represents string correlations of the grand canonical state $\hat{\rho}_{\mathrm{gc}}(\beta, \mu)$, with $\beta=0.788 \mathrm{~J}^{-1}$, and $\mu=0.475 U_{\mathrm{Q}}$. (b) Each line refers to a different length, ranging from $\ell=1$ (dark green) to $\ell=26$ (bright green), with spacing 1. (c), (d) Mean and variance of the particle number for $\hat{\rho}_{\mathrm{gc}}(\beta, \mu)$ as a function of $\mu$, for the $\beta$ matching the initial state energy: $0.578,0.622,0.67,0.724$, and $0.786 J^{-1}$, respectively.

using a quantum gas microscope [31]. Moreover, Ref. [11] reports an experimental study of the nonequilibrium dynamics of two-point correlations past a sudden quench with the same setup: Starting from a deep MI at $U_{0}=40 \mathrm{~J}$, the lattice strength is lowered to values of $U_{\mathrm{Q}}$ ranging between $5 \mathrm{~J}$ and $9 \mathrm{~J}$; time scales of up to $2.6 \hbar / J$ have been reached.

The $\left\langle\hat{\mathcal{O}}_{i, j}^{(\mathrm{BH})}\right\rangle(t)$ string propagation displays the same qualitative behavior of the spin-1 case (see Fig. 4, upper panels, for a typical situation). The interpretation of long-time properties in terms of a grand canonical ensemble $\hat{\rho}_{\mathrm{gc}}(\beta, \mu) \doteqdot$ $e^{-\beta\left(\hat{\mathcal{H}}_{\mathrm{BH}}-\mu \hat{N}\right)} / \mathcal{Z}^{\prime}\left(\hat{N} \doteqdot \sum_{j} \hat{n}_{j}\right)$ requires a careful analysis. We identify a region $\Omega$ in the $(\beta, \mu)$ plane where the energy and number of particles of $\hat{\rho}_{\mathrm{gc}}(\beta, \mu)$ match those of the initial MI up to $10^{-3}$ accuracy. The string properties of states inside $\Omega$ differ significantly, mainly because $\Omega$ comprises a large range of values for $\beta$. The lower panels of Fig. 4 show $\langle\hat{N}\rangle$ and $\left\langle\hat{N}^{2}\right\rangle-\langle\hat{N}\rangle^{2}$ for several states in the region.
The accuracy with which $\hat{\rho}_{\mathrm{gc}}(\beta, \mu)$ reproduces the long-time dynamics is nontrivially related to those expectation values, so that the employment of a thermal state with a fixed number of particles could be necessary to describe the dynamical data [51]. Interestingly, it is possible to find an optimal value $\left(\beta^{*}, \mu^{*}\right) \in \Omega$ reproducing $\left\langle\hat{\mathcal{O}}_{i, i+\ell}^{(\mathrm{BH})}\right\rangle(t \rightarrow \infty)$.

Finally, we address the specific physics of the bosonic Haldane insulator [7,35], the density-sector analog of the Haldane phase in integer spin systems. At present, two experimental challenges prevent its observation in a cold atomic gas: (i) the engineering of nearest-neighbor interactions; and (ii) the measurement of the appropriate string operator: $\hat{\mathcal{O}}_{i, j}^{(\mathrm{HI})} \doteqdot \delta \hat{n}_{i} \otimes e^{i \pi \delta \hat{n}_{i+1}} \cdots e^{i \pi \delta \hat{n}_{j-1}} \otimes \delta \hat{n}_{j}$ (the operator $\hat{n}_{i}$ has not been accessed yet). Long-range interactions can be realized via dressed Rydberg gases, polar molecules, or even atoms with a permanent magnetic dipole. The on-site occupation number could be detected by preparing a single 1D system and letting the atoms tunnel orthogonally before the detection. Finally, the presence of nonlocal order in other realistic cold-atom setups has been pointed out [52,53], which may provide novel observational platforms.

Conclusions. We presented a detailed study of the time evolution of a string operator past a sudden quantum quench. Such correlations signal a time-propagating horizon separating thermal from nonthermal regions. The presence of a duality mapping allowed for the formulation of a LR bound and for the interpretation of the data in terms of traveling quasiparticles. The investigation of thermalization properties for Hamiltonians where duality mappings cannot be established (which in our case do destroy SO abruptly and do not allow for the formulation of a LR bound) is an interesting problem left for a forthcoming publication. It is also intriguing to study SO and the other signatures of topological order for the Haldane phase (such as entanglement spectrum and edge modes) when protecting symmetries different from $\mathbb{D}_{2}$ are broken after the quench [32]. Our results can be tested experimentally in one-dimensional optical lattices in the Mott phase.

Acknowledgments. We thank P. Calabrese and V. Giovannetti for invaluable support. We also acknowledge enlightening discussions with E. Altman, M. C. Bañuls, M. Roncaglia, A. Silva, and L. Tagliacozzo. This work was supported by EU (IP-SIQS), by Italian MIUR via PRIN Project 2010LLKJBX and via FIRB Project RBFR12NLNA, and by Regione Toscana POR FSE 2007-2013. M.E. thanks Scuola Normale Superiore for hospitality.
[1] J. von Neumann, Z. Phys. 57, 30 (1929).

[2] J. M. Deutsch, Phys. Rev. A 43, 2046 (1991).

[3] M. Srednicki, Phys. Rev. E 50, 888 (1994).

[4] S. Åberg, Phys. Rev. Lett. 64, 3119 (1990); Ph. Jacquod and D. L. Shepelyansky, ibid. 79, 1837 (1997); G. Benenti, G. Casati, and D. L. Shepelyansky, Eur. Phys. J D 17, 265 (2001).

[5] X.-G. Wen, Quantum Field Theory of Many-Body Systems (Oxford University Press, New York, 2004).

[6] F. D. M. Haldane, Phys. Lett. A 93, 464 (1983); Phys. Rev. Lett. 50, 1153 (1983).
[7] E. G. Dalla Torre, E. Berg, and E. Altman, Phys. Rev. Lett. 97, 260401 (2006); E. Berg, E. G. Dalla Torre, T. Giamarchi, and E. Altman, Phys. Rev. B 77, 245119 (2008).

[8] T. Kinoshita, T. Wenger, and D. S. Weiss, Nature (London) 440, 900 (2006).

[9] S. Trotzky, Y.-A. Chen, A. Flesch, I. P. McCulloch, U. Schollwöck, J. Eisert, and I. Bloch, Nat. Phys. 8, 325 (2012).

[10] M. Gring, M. Kuhnert, T. Langen, T. Kitagawa, B. Rauer, M. Schreitl, I. Mazets, D. Adu Smith, E. Demler, and J. Schmiedmayer, Science 337, 1318 (2012). 
[11] M. Cheneau, P. Barmettler, D. Poletti, M. Endres, P. Schauß, T. Fukuhara, C. Gross, I. Bloch, C. Kollath, and S. Kuhr, Nature (London) 481, 484 (2012).

[12] T. Fukuhara, A. Kantian, M. Endres, M. Cheneau, P. Schauß, S. Hild, D. Bellem, U. Schollwöck, T. Giamarchi, C. Gross, I. Bloch, and S. Kuhr, Nat. Phys. 9, 235 (2013).

[13] T. Langen, R. Geiger, M. Kuhnert, B. Rauer, and J. Schmiedmayer, Nat. Phys. 9, 640 (2013).

[14] T. Fukuhara, P. Schauß, M. Endres, S. Hild, M. Cheneau, I. Bloch, and C. Gross, Nature (London) 502, 76 (2013).

[15] A. Polkovnikov, K. Sengupta, A. Silva, and M. Vengalattore, Rev. Mod. Phys. 83, 863 (2011).

[16] J. Dziarmaga, Adv. Phys. 59, 1063 (2010).

[17] A. Lamacraft and J. Moore, in Ultracold Bosonic and Fermionic Gases, edited by K. Levin, A. L. Fetter, and D. M. Stamper-Kurn (Elsevier, New York, 2012) Chap. 7, p. 177.

[18] M. Rigol, V. Dunjko, and M. Olshanii, Nature (London) 452, 854 (2008).

[19] M. Moeckel and S. Kehrein, Phys. Rev. Lett. 100, 175702 (2008).

[20] G. Roux, Phys. Rev. A 79, 021608 (2009).

[21] G. Biroli, C. Kollath, and A. M. Läuchli, Phys. Rev. Lett. 105, 250401 (2010).

[22] A. Pal and D. A. Huse, Phys. Rev. B 82, 174411 (2010).

[23] M. C. Bañuls, J. I. Cirac, and M. B. Hastings, Phys. Rev. Lett. 106, 050405 (2011).

[24] E. Canovi, D. Rossini, R. Fazio, G. E. Santoro, and A. Silva, Phys. Rev. B 83, 094431 (2011).

[25] G. P. Brandino, A. De Luca, R. M. Konik, and G. Mussardo, Phys. Rev. B 85, 214435 (2012).

[26] G. Carleo, F. Becca, M. Schiró, and M. Fabrizio, Sci. Rep. 2, 243 (2012).

[27] D. I. Tsomokos, A. Hamma, W. Zhang, S. Haas, and R. Fazio, Phys. Rev. A 80, 060302(R) (2009).

[28] A. Rahmani and C. Chamon, Phys. Rev. B 82, 134303 (2010).

[29] G. B. Halász and A. Hamma, Phys. Rev. Lett. 110, 170605 (2013).

[30] A. A. Patel, S. Sharma, and A. Dutta, Eur. Phys. J. B 86, 367 (2013).

[31] M. Endres, M. Cheneau, T. Fukuhara, C. Weitenberg, P. Schauss, C. Gross, L. Mazza, M. C. Bañuls, L. Pollet, I. Bloch, and S. Kuhr, Science 334, 200 (2011).

[32] F. Pollmann, A. M. Turner, E. Berg, and M. Oshikawa, Phys. Rev. B 81, 064439 (2010).
[33] F. Pollmann, E. Berg, A. M. Turner, and M. Oshikawa, Phys. Rev. B 85, 075125 (2012).

[34] M. den Nijs and K. Rommelse, Phys. Rev. B 40, 4709 (1989).

[35] D. Rossini and R. Fazio, New J. Phys. 14, 065012 (2012).

[36] I. Affleck, T. Kennedy, E. H. Lieb, and H. Tasaki, Phys. Rev. Lett. 59, 799 (1987); Commun. Math. Phys. 115, 477 (1988).

[37] A. Klümper, A. Schadschneider, and J. Zittartz, Europhys. Lett. 24, 293 (1993).

[38] U. Schollwöck, Ann. Phys. 326, 96 (2011).

[39] We compute the time evolution of chains up to $L=60$ sites with open-boundary conditions. The maximum allowed bond link is $D=2000$ and the truncation error is set to $10^{-10}$. Finite-size effects are under control and the analysis of the innermost 30 sites provides genuine bulk information.

[40] G. Vidal, Phys. Rev. Lett. 93, 040502 (2004); A. J. Daley, C. Kollath, U. Schollwöck, and G. Vidal, J. Stat. Mech. (2004) P04005.

[41] A. E. Feiguin and S. R. White, Phys. Rev. B 72, 220401(R) (2005).

[42] W. Chen, K. Hida, and B. C. Sanctuary, Phys. Rev. B 67, 104401 (2003).

[43] H. Ueda, H. Nakano, and K. Kusakabe, Phys. Rev. B 78, 224402 (2008).

[44] E. H. Lieb and D.W. Robinson, Commun. Math. Phys. 28, 251 (1972).

[45] S. Bravyi, M. B. Hastings, and F. Verstraete, Phys. Rev. Lett. 97, 050401 (2006).

[46] T. Kennedy and H. Tasaki, Phys. Rev. B 45, 304 (1992).

[47] M. Oshikawa, J. Phys.: Condens. Matter 4, 7469 (1992).

[48] P. Calabrese, F. H. L. Essler, and M. Fagotti, Phys. Rev. Lett. 106, 227203 (2011).

[49] Strictly speaking, because of the conservation of the total magnetization $\hat{M}^{z}=\sum_{i} \hat{S}_{i}^{z}$, one has to deal with a generalized Gibbs ensemble $e^{-\beta \hat{\mathcal{H}}-\lambda \hat{M}^{z}} / \mathcal{Z}^{\prime}$. However, since Hamiltonian (2) has particle-hole symmetry, $\lambda=0$ enforces the constraint $\left\langle\hat{M}^{z}\right\rangle=0$.

[50] D. Pérez-García, M. M. Wolf, M. Sanz, F. Verstraete, and J. I. Cirac, Phys. Rev. Lett. 100, 167202 (2008).

[51] A. C. Cassidy, C. W. Clark, and M. Rigol, Phys. Rev. Lett. 106, 140405 (2011).

[52] A. Montorsi and M. Roncaglia, Phys. Rev. Lett. 109, 236404 (2012).

[53] S. P. Rath, W. Simeth, M. Endres, and W. Zwerger, Ann. Phys. (N.Y.) 334, 256 (2013). 